\title{
Achievements and Challenges in Sensor Devices
}

\author{
Elisabetta Comini* \\ Sensor, Department of Information Engineering, University of Brescia, Brescia, Italy
}

Keywords: sensor device, sensor device and technology, nanomaterial, sensors, devices

A sensor device's purpose is to detect events or changes in the environment and send the information to its readout electronics or computer processors, meaning appropriate electronics are a fundamental requirement for sensor devices. In the early years, the sensing devices used to measure chemical, physical, or biological parameters were bulky. They were also often inaccurate, as the end user had to manually read and even decode the sensor signal.

Nanotechnological interdisciplinary advancements have triggered many recent advances in the sensing field, opening many new solutions for highly engineered devices with excellent performance characteristics.

Sensors play a central role in the improvements necessary to meet social demands, such as hazard detection (Rasheed et al., 2018), pollution problems and environmental remediation (Shak et al., 2018), energy production (Hou et al., 2018) and storage (Kawai et al., 2018), and biomedical treatments (Kumar and Liz-Marzán, 2019).

They may be roughly classified according to the phenomena that needs to be sensed: chemical substances, physical conditions, or biological phenomena.

Their variety has been steadily increasing over the years, and among them we have:

OPEN ACCESS

Edited and reviewed by: Dermot Diamond,

Dublin City University, Ireland

*Correspondence: Elisabetta Comini elisabetta.comini@unibs.it

Specialty section:

This article was submitted to Sensor Devices,

a section of the journal

Frontiers in Sensors

Received: 16 September 2020 Accepted: 05 October 2020

Published: 11 February 2021

Citation:

Comini E (2021) Achievements and

Challenges in Sensor Devices.

Front. Sens. 1:607063.

doi: 10.3389/fsens.2020.607063
- Magnetic sensors

- Photonics based sensors

- Infrared sensors

- Gas Sensors

- Geo-sensors

- Touch sensing devices

- Nano-sensors

- Clothes-based sensors

- Crowd sensing devices

- Sensors decoding physical sensation

- Movement sensors

- Atmosphere sensors

- Position sensors

- Liquid sensors

- Sound sensors

- Medical sensing devices

Social demands require an enhancement in key sensor performance characteristics such as sensitivity, selectivity, stability, and usability. Therefore, research has focused on advancing the design of sensing mechanisms and developing new active sensing materials. Nanotechnology and material science play a key role in this regard and are continuously pursuing innovations for improved sensor devices. 
In recent years, smart, safe, biocompatible, and environmentally "clean" sensor devices and instruments have been designed and developed. These sensor devices and instruments embrace multiple integrated functionalities, including wearable electronics, smartphones, and other commoditized gadgets. Through these technology platforms, different parameters/phenomena can now be monitored simultaneously, and new technologies and sensor types have been proposed.

Due to the advanced properties of many electronic sensor devices, including their small dimensions, low weight and cost, and reliability, they are strongly aligned with various markets. Optical sensor devices have lately been gaining interest, especially for specific applications in critical environmental applications (pipelines, power lines, perimeters, borders, etc.), due to their ability to reach places that are otherwise inaccessible. Optical fibers can monitor strain, temperature, pressure, and vibrations at millions of points, increasing the monitoring scalability capabilities of sensing devices (Fernández-Ruiz et al., 2020).

The limits of sensing devices in terms of sensitivity, selectivity, resolution, accuracy, and precision are continuously being improved. At the same time, their potential in terms of exploitation and applications is also rapidly expanding.

Simultaneously, we have witnessed the advent of the Internetof-Things (IoT) that has changed the way we think about sensors and their usage, and their diffusion throughout society. IoT is a network of "intelligent" devices with embedded electronics, sensors, and network connectivity that can acquire and exchange data. IoT concepts and technologies have been implemented extensively in automobiles and have been used to create smart lighting in homes and streets and network water, power, temperature control, and alarm systems in cities (Vlacheas et al., 2013; Jin et al., 2014; Zanella et al., 2014; Zhu et al., 2015).

In this IoT era, the biggest challenge for any device is providing data in real time so that the status of key parameters can be tracked, and exchanging this information with other devices while learning the overall functionality of the system, therefore acting even beyond their sensing function.

In recent years, particular attention has been devoted to wearable sensing devices, especially concerning fitness applications and IoT. The advent of smart-watches and wristbands now allows activities throughout the day to be monitored without creating discomfort for the person (Haghi et al., 2017). Lately, wearable sensing capabilities have been spreading to smart textiles, clothes with embedded electronics, and smart spectacles, increasing the number of parameters that can be monitored, from which patterns and trends can be extracted, to which personalized conditioning or care strategies can be optimized (Stavropoulos et al., 2017).

For example, research has led to innovations based on flexible and stretchable electronic devices, particularly for medical applications, such as employing novel materials and devices that have properties similar to human tissue (Wang et al., 2018; Lee et al., 2019; Niu et al., 2019; Yang et al., 2020). This in turn has widened the field of wearable bioelectronics and biosensors devices thanks to their increasing bio-integration with human tissues.
Such devices may monitor the electrical properties of tissues, providing appropriate feedback, real-time diagnosis, and therapies based on "closed loop" treatment. Some examples of flexible and stretchable devices are electronic skins (e-skin) (Hanif et al., 2018; Park et al., 2019), glucose monitoring contact lenses (Kim et al., 2017; Pakr et al., 2018) and wearable health monitors (Hong et al., 2018; Xie et al., 2020).

In terms of well-being and healthcare, IoT may allow continuous and holistic monitoring thanks to sensors interacting with the environment, offering autonomous communication and providing contextual information to assist with clinical decisionmaking and supporting caregivers, especially with respect to care for the elderly.

Especially during this sanitary emergency period of COVID19 , it is evident that sensors that can provide accurate, rapid information have a vital role to play in reducing the spread of viruses and infections, thereby saving numerous lives.

Researchers in this field are therefore continuously looking for more sensitive and selective detection strategies and measuring principles and new analytical methodologies to develop modern sensing devices and instruments. From a technological point of view, the main challenges of sensor devices are related to the reduction of their cost, size, and energy consumption. Moreover, additional efforts in design and development of nanoscale sensing materials have to be made to achieve improved device performance. Another challenge, sometimes disregarded and underestimated, is the reliable integration of these novel materials and structures into sensor devices. Their integration must be scalable for the production of commercial devices, as their use will be otherwise limited to laboratory experiments, with very limited socio-economic impact.

Improvements in sensing devices' performance may be achieved by the exploitation of $0 \mathrm{D}, 1 \mathrm{D}$, and $2 \mathrm{D}$ materials together with flexible and bio-inspired concepts. Concerning their integration, top down technology (conventionally used for microfabrication) must be integrated synergistically with bottom up advances (mostly used for nanomaterials fabrication) for the combination of nanomaterials at a sensor device scale, allowing their full exploitation.

Another important aspect of sensors, that is sometimes not considered as part of the sensing field, is that they produce data. These data must be processed and decoded, and this is an active part of research in sensor technology. The sensed data needs to be analyzed and transformed to provide the final feedback to the end user in real time, using artificial intelligence, deep learning, or other techniques to manage "big data." Managing these increasingly large data sets streaming from highly distributed and heterogeneous sources is a rapidly growing challenge in this field.

It is extremely difficult to predict the real-time evolution due to all the variables involved in this multidisciplinary field of sensing devices, but significant advances have been made that have had a huge impact on society, improving product quality, food and environment safety, disease diagnosis, medicine, health and wealth, process studies, and more.

We can be certain that this trend will continue as new sensor technologies emerge and broaden the scale and scope of impact on our lives. 
"Frontiers in Sensors" will offer a forum for the publication of quality research, and for informed discussion on trends and opportunities for Sensor Devices in particular, to deliver a significant positive socio-economic impact in the coming years.

\section{REFERENCES}

Fernández-Ruiz, M. R., Soto, M. A., Williams, E. F., Martin-Lopez, S., Zhan, Z., Gonzalez-Herraez, M., et al. (2020). Distributed acoustic sensing for seismic activity monitoring. APL Photonics 5:030901. doi: 10.1063/1.5139602

Haghi, M., Thurow, K., and Stoll, R. (2017). Wearable devices in medical internet of things: scientific research and commercially available devices. Healthc. Inform. Res. 23:24. doi: 10.4258/hir.2017.23.1.4

Hanif, A., Trung, T. Q., Siddiqui, S., Toi, P. T., and Lee, N.-E. (2018). A stretchable strain-insensitive temperature sensor based on free-standing elastomeric composite fibers for on-body monitoring of skin temperature. ACS Appl. Mater. Interfaces 10:27297. doi: 10.1021/acsami.8b19425

Hong, Y. J., Lee, H., Kim, J., Lee, M., Choi, H. J., Hyeon, T., et al. (2018). Multifunctional wearable system that integrates sweat-based sensing and vitalsign monitoring to estimate pre-/post-exercise glucose levels. Adv. Funct. Mater. 28:1805754. doi: 10.1002/adfm.201805754

Hou, J., Inganäs, O., Friend, R. H., and Gao, F. (2018). Organic solar cells based on non-fullerene acceptors. Nat. Mater. 17, 119-128. doi: 10.1038/nmat5063

Jin, J., Gubbi, J., Marusic, S., and Palaniswami, M. (2014). An information framework for creating a smart city through internet of things. IEEE Intern. Things J. 2, 112-121. doi: 10.1109/JIOT.2013.2296516

Kawai, T., Nakao, S., Nishide, H., and Oyaizu, K. (2018). Poly(diphenanthrenequinone-substituted norbornene) for long life and efficient lithium battery cathodes. Bull. Chem. Soc. Jpn. 91, 721-727. doi: 10.1246/bcsj.20170420

Kim, J., Kim, M., Lee, M.-S., Kim, K., Ji, S., Kim, Y.-T., et al. (2017). Wearable smart sensor systems integrated on soft contact lenses for wireless ocular diagnostics. Nat. Commun. 8:14997. doi: 10.1038/ncomms 14997

Kumar, J., and Liz-Marzán, L. M. (2019). Recent advances in chiral plasmonics - towards biomedical applications. Bull. Chem. Soc. Jpn. 92, 30-37. doi: 10.1246/bcsj.20180236

Lee, J., Cho, H. R., Cha, G. D., Seo, H., Lee, S., Park, C.-K., et al. (2019). Flexible, sticky, and biodegradable wireless device for drug delivery to brain tumors. Nat. Commun. 10:5205. doi: 10.1038/s41467-019-13198-y

Niu, S., Matsuhisa, N., Beker, L., Li, J., Wang, S., Wang, J., et al. (2019). A wireless body area sensor network based on stretchable passive tags. Nat. Electron. 2:361. doi: 10.1038/s41928-019-0286-2

Pakr, J., Kim, J., Kim, S.-Y., Cheong, W. H., Jang, J., Park, Y.-G., et al. (2018). Soft, smart contact lenses with integrations of wireless circuits, glucose sensors, and displays. Sci. Adv. 4:9841. doi: 10.1126/sciadv.aap9841

Park, J., Lee, Y., Barbee, M. H., Cho, S., Cho, S., Shanker, R., et al. (2019). A hierarchical nanoparticle-in-micropore architecture for enhanced

\section{AUTHOR CONTRIBUTIONS}

The author confirms being the sole contributor of this work and has approved it for publication.

mechanosensitivity and stretchability in mechanochromic electronic skins. Adv. Mater. 31:1808148. doi: 10.1002/adma.201808148

Rasheed, T., Bilal, M., Nabeel, F., Iqbal, H. M. N., Li, C., and Zhou, Y. (2018). Fluorescent sensor based models for the detection of environmentally-related toxic heavy metals. Sci. Total Environ. 615, 476-485. doi: 10.1016/j.scitotenv.2017.09.126

Shak, K. P. Y., Pang, Y. L., and Mah, S. K. (2018). Nanocellulose: recent advances and its prospects in environmental remediation. Beilstein J. Nanotechnol. 9, 2479-2498. doi: 10.3762/bjnano.9.232

Stavropoulos, T. G., Meditskos, G., and Kompatsiaris, I. (2017). DemaWare 2: integrating sensors, multimedia and semantic analysis for the ambient care of dementia. Pervasive Mob. Comput. 34, 126-145. doi: 10.1016/j.pmcj.2016.06.006

Vlacheas, P., Giaffreda, R., Stavroulaki, V., Kelaidonis, D., Foteinos, V., Poulios, G., et al. (2013). Moessner, enabling smart cities through a cognitive management framework for the internet of things. IEEE Commun. Mag. 51, 102-111. doi: 10.1109/MCOM.2013.6525602

Wang, C. H., Li, X. S., Hu, H. J., Zhang, L., Huang, Z. L., Lin, M. Y., et al. (2018). Monitoring of the central blood pressure waveform via a conformal ultrasonic device. Nat. Biomed. Eng. 2:687. doi: 10.1038/s41551-018-0287-x

Xie, Z., Avila, R., Huang, Y., and Rogers, J. A. (2020). Flexible and stretchable antennas for biointegrated electronics. Adv. Mater. 32:1902767. doi: 10.1002/adma.201902767

Yang, Y., Song, Y., Bo, X., Min, J., Pak, O. S., Zhu, L., et al. (2020). A laser-engraved wearable sensor for sensitive detection of uric acid and tyrosine in sweat. Nat. Biotechnol. 38:217. doi: 10.1038/s41587-019-0321-x

Zanella, A., Bui, N., Castellani, A., Vangelista, L., and Zorzi, M. (2014). Internet of things for smart cities. IEEE Intern. Things J. 1, 22-32. doi: 10.1109/JIOT.2014.2306328

Zhu, C., Leung, V. C. M., Shu, L., and Ngai, E. (2015). Green internet of things for smart world. IEEE Access 3, 2151-2162. doi: 10.1109/ACCESS.2015.2497312

Conflict of Interest: The author declares that the research was conducted in the absence of any commercial or financial relationships that could be construed as a potential conflict of interest.

Copyright (C) 2021 Comini. This is an open-access article distributed under the terms of the Creative Commons Attribution License (CC BY). The use, distribution or reproduction in other forums is permitted, provided the original author(s) and the copyright owner(s) are credited and that the original publication in this journal is cited, in accordance with accepted academic practice. No use, distribution or reproduction is permitted which does not comply with these terms. 